\title{
Correlating Dementia Severity Rating Scale and Schonberg Index Mortality Risk Scores to Aid Caregiver Medical Decision-Making Myranda Grismore, BS ${ }^{1}$, Nicole Fowler, PhD, MHSA ${ }^{1,2,3}$ \\ ${ }^{1}$ Indiana University School of Medicine, ${ }^{2}$ Indiana University Center for Aging Research, ${ }^{3}$ Regenstrief Institute
}

\section{Background/Hypothesis:}

The 16 million unpaid caregivers serving individuals with Alzheimer's disease and related dementias (ADRD) are more likely to participate in medical decision-making than caregivers of older adults with other chronic conditions. Their decisions may reflect the patient's severity of cognitive impairment and life expectancy. Analysis of the relationship between these two factors is needed to improve the prognostic information provided to caregivers. ADRD severity can be measured using the Dementia Severity Rating Scale (DSRS), a validated, informant-based multiple-choice questionnaire that assesses functional and cognitive abilities. Life expectancy can be estimated using the Schonberg Index (SI), a validated, predictive mortality index. We hypothesize that DSRS and SI scores will be correlated.

\section{Methods:}

A retrospective analysis of data from 230 dyads enrolled in the Decisions about Cancer Screening in Alzheimer's Disease (DECAD) study was performed. The DECAD study is an ongoing randomized controlled trial enrolling dyads of older women with ADRD and their caregivers to test the benefits of a mammography decision aid. In the study, caregivers completed the DSRS and SI questions. DSRS and SI scores were compared using linear regression analysis. Additionally, 20 DECAD interviews were qualitatively evaluated to explore themes of medical decision-making among ADRD caregivers.

\section{Results:}

DSRS scores and SI scores were weakly correlated, yielding an $\mathrm{R}^{2}$ value of 0.1 . Interviews revealed that caregivers most frequently considered physician advice, comorbidity status, and patient preferences in decision-making. $67 \%$ of moderate ADRD caregivers and $36 \%$ of mild ADRD caregivers cited cognitive impairment severity as an influential factor.

\section{Conclusions/Implications:}

Further analysis controlling for time since diagnosis will better establish the relationship between DSRS and SI scores. The resulting increase in accuracy of ADRD prognostic information will aid physicians and caregivers in medical decision-making. Furthermore, understanding what influences caregiver decisions will allow for the provision of improved guidance from physicians. 